\title{
LA VALORACIÓN PATRIMONIAL DEL PAISAJE CULTURAL CAFETERO DEL TOLIMA, COLOMBIA. RETOS Y ESTRATEGIAS PARA SU CONSERVACIÓN Y APROPIACIÓN.
}

\author{
César Augusto Velandia Silva \\ Universidad de Ibagué, Facultad de Humanidades, Artes y Ciencias Sociales. Programa de Arquitectura. \\ Cesar.velandia@unibague.edu.co
}

\section{RESUMEN}

El proyecto de investigación "Paisaje Cultural Cafetero del Tolima", pretende identificar el paisaje cultural como bien de interés cultural de esta región en Colombia. Se propone el desarrollo de una puesta en valor de los atributos del paisaje y de sus enlaces naturaleza-cultura. Primero, debido a su pertinencia para el conocimiento y la apropiación de la cultura regional y segundo, debido a que la investigación científica aplicada a una región de alta calidad productiva agrícola a nivel nacional, es desconocida.

Lo anterior permitirá formular estrategias en atención a las implicaciones del paisaje ante el ordenamiento territorial, la variabilidad climática, la sostenibilidad ambiental, la gestión del riesgo, las actividades turísticas y el relevo generacional de jóvenes que ya no quieren ser caficultores. Asimismo, el paisaje cultural se muestra, como el escenario idóneo para acoger los procesos de cohesión y reconstrucción del tejido social de las comunidades en el marco de la Paz.

Palabras Clave: paisaje cultural, arqueogeografía, patrimonio.

\begin{abstract}
The research project "Coffee Cultural Landscape of Tolima", aims to identify the cultural landscape as a cultural asset in the Tolima region, Colombia. It proposes the development of an appraisement project about the attributes of the landscape and its culture-nature linkages. First, because it is relevant to the knowledge and appropriation of the regional culture of Tolima, and second, because scientific research applied to a region of high productive agricultural quality at the national level, is unknown.

This will enable the formulation of strategies attending implications for land use, climate change, environmental sustainability and risk management, also tourism activities and generational change of thousands of youngsters who no longer want be coffee growers. Likewise, the cultural landscape is shown, as the ideal setting to embrace the processes of cohesion and reconstruction of the social linkages of the communities within the framework of Peace.
\end{abstract}

Key words: cultural landscape, heritage, culture-nature linkages 


\section{LOS ANTECEDENTES}

La iniciativa de identificar y delimitar el paisaje cultural cafetero de la región del Tolima estuvo considerada dentro del proyecto del expediente de nominación del Paisaje Cultural Cafetero de Colombia (PCCC) ante Unesco; sin embargo, durante el proceso, ésta no quedó incluida en la delimitación del bien patrimonial inscrito en la Lista de Patrimonio Mundial en 2011.

A partir de este evento, el gobierno colombiano emprendió una serie de acciones coordinadas con la Federación Nacional de Cafeteros y las entidades regionales (51 municipios en los departamentos de Caldas, Risaralda, Quindío y Valle del Cauca), con objeto de promover acciones para la conservación del sitio.

Esta experiencia, sin precedente en el ámbito colombiano hasta el año 2011, ha servido para establecer nuevas referencias sobre la valoración patrimonial del paisaje, y atender los retos que representan la difusión, la coordinación y la gestión del paisaje cultural; y aplicarlas a los sitios patrimoniales de Colombia.

Por tanto, se considera fundamental contar con una puesta en valor que resalte los atributos del paisaje cultural cafetero del Tolima (PCCT), y su alto potencial cultural, en conocimiento comparativo de posibles valores excepcionales similares al PCCC. La puesta en valor de una referencia cultural histórica tan importante como el café del Tolima, puede apoyar el proceso de la Visión Tolima y los macroproyectos planteados. Tal es el caso de "Tolima, primer productor de café de alta calidad en Colombia". ${ }^{15}$

Este macroproyecto, puede fortalecerse de forma integral con el aspecto productivo e institucional si se apoya con un proceso de conocimiento que potencie la relación que los habitantes de la región tienen en mente sobre la identidad cafetera, pero que, por no contar con una producción intelectual adecuada, no se difunde ni promueve de forma institucional en todos los niveles, sea privado, público, individual o colectivo.

Por fortuna, el Comité Departamental de Cafeteros del Tolima obtuvo la Denominación de Origen Café de Tolima, una de las seis denominaciones otorgadas a nivel nacional; lo que ratifica el café del Tolima como uno de los mejores de Colombia; lo constituye un atributo que motiva la necesidad de valorar la cultura del café de la región.

Localizada en la cuenca del Río Magdalena, paralela a la cuenca del Cauca (la del PCCC), el PCCT, es en una de sus partes más representativas, un espejo del paisaje cultural inscrito; el territorio en el que se presentan los enlaces naturalezacultura posibilitan la articulación de los elementos tangibles e intangibles manifestados en la biodiversidad, la arquitectura, el urbanismo, la arqueología, las cocinas tradicionales, los cantos, la poesía, los saberes, entre otros atributos.

Adicionalmente, la valoración del paisaje cultural requiere desarrollar un conocimiento sobre los retos que le presenta el territorio del Tolima en términos de la gestión de riesgo y amenaza del Volcán Cerro Machín (VCM), y el Volcán Nevado del Ruiz (VNR); los Planes de Ordenamiento Territorial (POT) y el Plan de Ordenamiento Departamental (POD); el impacto de la variabilidad climática en la productividad; la sostenibilidad ambiental ante el impacto de las actividades turísticas y la conservación de la biodiversidad; el relevo generacional y el proceso de instauración de la Paz.

15 http://www.visiontolima2025.org/index.php/ideas-fuerza/macroproyectos/60-macroproyectos 


\section{LA BASE METODOLÒGICA}

La base metodológica para la formulación del proyecto se fundamenta en la elaboración de un marco teórico-conceptual, la definición del problema respaldada por el planteamiento de las preguntas de investigación y la identificación de los objetivos.

\subsection{El marco teórico-conceptual}

Por su colindancia, el proyecto contiene una referencia directa con los retos y oportunidades que ha presentado la experiencia del Paisaje Cultural Cafetero de Colombia (PCCC), inscrito en la Lista del Patrimonio Mundial de la UNESCO en 2011, el mayor paisaje cultural productivo de América Latina.

El concepto de paisaje se refiere a la complejidad del territorio donde los aspectos culturales, sociales y productivos se encuentran con la biodiversidad. A partir del hecho que el PCCC es una muestra de un paisaje único, productivo y sostenible y que representa la tradición cafetera colombiana; el paisaje cafetero, per se, es la manifestación de los enlaces naturaleza-cultura representados en una tradición centenaria, ilustrada por la adaptación los minifundios, el uso inteligente de las fuentes de agua, el desarrollo de materiales tradicionales de construcción, la comprensión simbólica del paisaje y la coexistencia con el riesgo de desastres.

El 25 de junio de 2011, el Comité del Patrimonio Mundial de la Unesco en su 35a reunión, inscribió al PCCC en la Lista del Patrimonio Mundial. El PCCC fue inscrito debido al uso continuo de la tierra donde los agricultores aplicaron prácticas de manejo innovadoras de los recursos naturales y produjeron manifestaciones culturales tangibles e intangibles que caracterizan a la región.

El paisaje cultural es una manifestación territorial del patrimonio, es decir de las prácticas tradicionales heredadas a través del tiempo, de una región y sus comunidades. También denota la evolución, el desarrollo y el cambio en el tiempo, de su relación con su entorno natural, cultural y económico. En América Latina existen sólo tres paisajes culturales:

i) El Paisaje arqueológico de las primeras plantaciones de café del sureste de Cuba donde los restos únicos de las plantaciones de café del siglo XIX, en las estribaciones de la Sierra Maestra, muestran evidencia de una forma pionera de la agricultura en las dificultades del terreno; ii) El Paisaje del agave y las antiguas instalaciones industriales de tequila en México donde un amplio paisaje de agaves azules, utilizado desde el siglo XVI para producir tequila, hoy representa la identidad nacional. Este paisaje incluye los restos arqueológicos de cultivos en terrazas, casas, templos, túmulos ceremoniales y juegos tradicionales; y iii) El Paisaje Cultural Cafetero de Colombia, basado en criterio v y vi de Unesco.

Los criterios son: $(v)$ "por ser un ejemplo excepcional de asentamiento humano tradicional, uso de la tierra, o el uso de mar, que es representativo de una cultura -o culturas-, o de la interacción humana con el medio ambiente, especialmente cuando se ha vuelto vulnerable debido al impacto de irreversible cambio, y (vi) estar directa o materialmente asociado con acontecimientos o tradiciones vivas, con ideas, o con creencias, con obras artísticas y literarias de significado universal excepcional." 16

En el caso de Colombia, adicionalmente cuenta con procesos de formulación de paisajes culturales, que forman parte de la Lista Tentativa de Unesco ${ }^{17}$ y que han desarrollado metodologías aplicadas para la delimitación, identificación, valoración y manejo de los siguientes sitios: el paisaje de la infraestructura de la United Fruit Company, el paisaje cultural

\footnotetext{
16 Unesco, The Criteria for Selection. http://whc.unesco.org/en/criteria/

17 http://whc.unesco.org/en/tentativelists/state $=$ co 
de los Pueblos de la Sal; el paisaje del sur de la Provincia de Ricaurte y el paisaje de la cuenca baja del Río Chicamocha. (Fundación Erigaie, 2010).
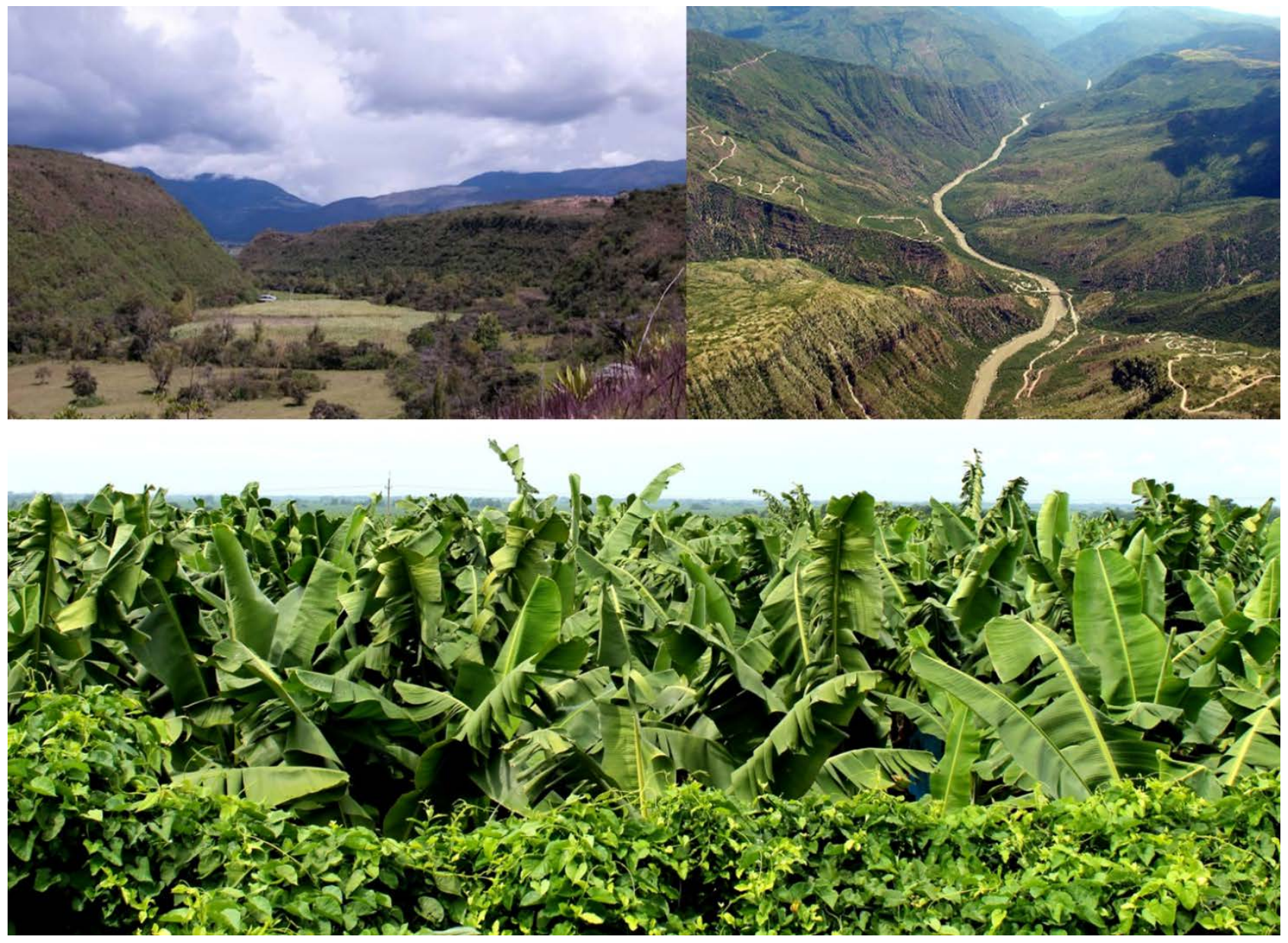

Fig. 01. Paisaje de la infraestructura de la United Fruit Company; paisaje del sur de la Provincia de Ricaurte y el paisaje de la cuenca baja del Río Chicamocha. Fuente: Ministerio de Cultura de Colombia.

La categoría de Paisajes Culturales en el Patrimonio Mundial fue adoptada por el Comité de Patrimonio Mundial durante su $16^{\circ}$ Sesión, llevada a cabo en Santa Fe, Estados Unidos de América, entre el 7 y el 14 de diciembre de 1992; la Convención del Patrimonio Mundial se convirtió en el primer instrumento legal internacional en reconocer y proteger los paisajes culturales. Asuntos relacionados con la mixtura del patrimonio cultural y natural y los vínculos intrínsecos entre las comunidades y su entorno natural fueron discutidos desde la adopción de la Convención de Patrimonio Mundial en 1992.

En las Directrices Practicas para la implementación de la Convención del Patrimonio Mundial (UNESCO, 2008) se señala que "Ios paisajes culturales son bienes culturales y representan las "obras conjuntas del hombre y la naturaleza. Ilustran la evolución de la sociedad humana y sus asentamientos a lo largo del tiempo, condicionados por las limitaciones y/o oportunidades físicas que presenta su entorno natural y por las sucesivas fuerzas sociales, económicas y culturales, tanto externas como internas". Así mismo, claramente se determina que los paisajes culturales se dividen en tres categorías principales: 
El más fácil de identificar es el paisaje claramente definido, concebido y creado intencionalmente por el hombre. Comprende los paisajes de jardines y parques creados por razones estéticas, que con frecuencia (pero no siempre) están asociados a construcciones o a conjuntos religiosos o monumentales.

La segunda categoría es la del paisaje que ha evolucionado orgánicamente. Es fruto de una exigencia originalmente social, económica, administrativa y/o religiosa y ha alcanzado su forma actual por asociación y, como respuesta a su entorno natural. Estos paisajes reflejan este proceso evolutivo en su forma y su composición. Se subdividen en dos categorías:

a) Un paisaje relicto (o fósil) es aquel que ha experimentado un proceso evolutivo que se ha detenido en algún momento del pasado, ya sea bruscamente o a lo largo de un periodo. Sus características esenciales siguen siendo, empero, materialmente visibles;

b) Un paisaje vivo es el que conserva una función social activa en la sociedad contemporánea, estrechamente vinculada al modo de vida tradicional, y en el cual prosigue el proceso evolutivo. Al mismo tiempo, presenta pruebas materiales manifiestas de su evolución en el transcurso del tiempo.

La última categoría comprende el paisaje cultural asociativo. La inscripción de este tipo de paisaje en la Lista del Patrimonio Mundial se justifica por la fuerza de evocación de asociaciones religiosas, artísticas o culturales del elemento natural, más que por huellas culturales tangibles, que pueden ser insignificantes o incluso inexistentes. (Rössler, M., 1993, 2003, 2009).

Sin embargo, en congruencia con la polisemia inherente al término "paisaje" y la connotación cultural del mismo, la pregunta que conviene, para comprender e interpretar con mayor profundidad, es ¿qué es lo cultural del paisaje?

A partir del legado de Sauer entendemos paisaje cultural como un registro del hombre sobre el territorio; un texto que se puede escribir e interpretar; interpretando el territorio como una construcción humana. Definimos paisaje cultural como un ámbito geográfico asociado a un evento, a una actividad o a un personaje histórico, que contiene valores estéticos y culturales. Dicho de una manera menos ortodoxa, pero más sencilla y hermosa, paisaje cultural es la huella del trabajo sobre el territorio, un memorial al trabajador desconocido." (Sabaté, J., 2010:11)

Anschuetz (2001) reproduce el paradigma del paisaje ${ }^{18}$ : “Paisaje no es sinónimo de medio ambiente. Los paisajes son sintéticos (Jackson, J., 1984:156). Los sistemas culturales estructuran y organizan las interacciones entre las gentes y su medio ambiente (Deetz, J., 1990; Ingold, T., 1993, Tuan, Y., 1977) Como observa Cosgrove (1985), "paisaje significa un mundo exterior mediatizado por la experiencia subjetiva del hombre" El paisaje es el mediador entre naturaleza y cultura. "Los paisajes son un mundo de productos culturales [...] un paisaje es "una construcción, una composición del mundo". (Boonemaison, J., 1994; Norton, W., 1989).

\footnotetext{
18 "Paisaje está relacionado con landscipe, que a su vez es un término que apareció a finales del siglo XVI de la palabra holandesa landschap (paisaje en alemán) para denotar una foto de un paisaje donde 'se deriva SCHAP' de la misma raíz que origina 'barco' y también está en shape (forma). Por último, la forma, el patrón y la relación son internas al propio concepto de 'paisaje': considerar un paisaje significa decodificar la forma del espacio en él." (F. Criado-Boado, 2014:6).
} 
Los paisajes son construcciones dinámicas en los que cada comunidad y cada generación impone sus propio mapa cognitivo de un mundo, antropogénico e interconectado, de morfología, planificación y significado coherente [...] Más aun, el paisaje, como sistema de manejo de símbolos significantes de las acciones humanas, y de los subproductos materiales que generan ayuda a definir relaciones habituales ayuda a definir relaciones habituales basadas en una información diferenciada. Sin embargo, los procesos de cambio conductual a través del tiempo y el espacio tienen forzosamente como resultado un paisaje en cambio constante. Así que el paisaje es un proceso cultural. [Hirsch, E. y O'Hanlon M., 1995] (Anschuetz, K., 2001:5).

El primer paisaje cultural inscrito en la Lista del Patrimonio Mundial en $1993^{19}$ fue el Parque Nacional Tongariro (Nueva Zelanda). Ésta nueva categoría ha permitido el reconocimiento del carácter no monumental del patrimonio integral en los paisajes culturales; el reconocimiento de los vínculos existentes entre diversidad cultural y biológica, específicamente con el uso sostenible de la tierra. (Bandarin, F., et al., 2009:3).

La Lista del Patrimonio Mundial cuenta con 99 paisajes culturales inscritos; 4 de ellos son trasfronterizos; 1 ha sido excluido de la Lista; 3 están en la Lista del Patrimonio Mundial en Peligro; 90 se han inscrito como sitios culturales y 9 se han inscrito como sitios mixtos. Los Paisajes Culturales inscritos en la Lista del Patrimonio Mundial de la Unesco se encuentran localizados en los territorios de 61 Estados Parte. ${ }^{20}$ Un análisis de las listas indicativas reveló que el $10 \%$ de los sitios incluidos en las Listas indicativas nacionales son sitios de importancia agrícola y pastoral. (Rössler, M., Tornoux M., 2013:13).

Es importante resaltar, el énfasis sobre el estudio de los enlaces naturaleza-cultura. Éstos se refieren a la identificación de la relación de los aspectos vivos vinculantes entre la dimensión natural y la dimensión cultural; concentradas en lo más profundo de los valores patrimoniales del sitio, las tradiciones simbólicas y su diversidad biocultural. Sobre la cual se "han forjado identidades culturales en entornos específicos [...] y la aprehensión del patrimonio tanto en el campo natural como en el campo cultural que ha evolucionado [...] como algo dinámico, interrelacionado y complejo." (Larsen, P., Wijesuriya, G., 2015:6.)

El paisaje como un modelo "vivo", se refiere a su comprensión como una expresión viva que debe permanecer expuesta para la aprehensión social y el desarrollo de las acciones para su preservación a través del tiempo. Esto es muy significativo en el paisaje agrícola: para una integración efectiva del patrimonio y el desarrollo sostenible, es necesario explorar sistemáticamente las características de complejidad de la naturaleza y la cultura para establecer un diálogo creativo entre el pasado, el presente y el futuro.

\subsection{Los problemas planteados}

La definición del problema radica en que no se cuenta en la región del Tolima con un conocimiento formado en función de la valoración de los atributos del paisaje cultural. La falta de líneas de investigación sobre el tema, no ha permitido el desarrollo de una construcción colectiva alrededor de la identidad cafetera y por tanto de la apropiación de la cultura regional del Tolima.

Constituida en la actualidad como una de las regiones de más alta calidad productiva agrícola y cafetera a nivel nacional, la propuesta del paisaje cultural cafetero del Tolima se presenta como una oportunidad de articulación de la dimensión cultural del territorio y permitir un mejoramiento de la calidad del desarrollo rural y de disminución de la problemática socioeconómica de las comunidades de la región.

20 http://whc.unesco.org/en/list/ 
El desarrollo del proyecto tendrá como alcance la publicación de un expediente de valoración del Paisaje Cultural Cafetero del Tolima (PCCT) como Bien de Interés Cultural (BIC), en el cual se propongan las estrategias para la conservación y la apropiación del PCCT como BIC departamental. En consecuencia, las preguntas de investigación apuntan a la definición de los atributos y valores del PCCT.

a) ¿Existen los mismos atributos del PCCC o se pueden identificar nuevos atributos en el PCCT?

b) ¿Cómo se presentan en el PCCT los factores que afectan la posible pérdida o amenaza del PCCC?

c) ¿Es factible la incorporación de criterios para la redefinición de los valores y atributos en función de los elementos no contenidos en la nominación del PCCC?

d) ¿Cuáles son las características excepcionales de las manifestaciones culturales materiales e inmateriales del PCCT?

e) ¿De qué manera afecta al PCCT el cambio climático y la gestión de amenaza y riesgo de desastres?

f) ¿El paisaje cafetero del sur del Tolima contiene nuevos atributos excepcionales diferenciados a los del PCCC?

\subsection{Los objetivos}

El proyecto tiene como objetivo general, identificar y delimitar el PCCT como Bien de Interés Cultural (BIC), en el cual deben proponerse las acciones tendientes a la conservación, la apropiación y la promoción del PCCT como BIC departamental; y definir las implicaciones y compromisos que esto conlleva en la educación, el ordenamiento territorial, las actividades extractivas, las actividades turísticas y en el relevo generacional de miles de jóvenes que no quieren ser caficultores. Asimismo, visualizar el paisaje cultural como escenarios aptos para la cohesión de las comunidades en la preservación de la cultura y la paz.

\section{EL CAFÉ DE LA REGIÒN DEL TOLIMA}

Guhl (2008:133), identifica que las condiciones ideales -y actuales- para la producción cafetera están entre los 1.000 y $2.000 \mathrm{msnm}$. Dado que el régimen de precipitación presenta una distribución bimodal por la ubicación ecuatorial, esto produce dos cosechas de café anuales. El café se cultiva por lo general en laderas muy empinadas, y casi siempre acompañado de otras actividades agropecuarias en la 'finca' ${ }^{21}$. Lo que produce un paisaje de 'colcha de retazos', en el que las parcelas de café se intercalan con potreros, cultivos de verduras y legumbres ${ }^{22}$, guaduales y bosques de galería entre otros.

"El café llegó al país por Venezuela, a la zona que corresponde hoy a los departamentos de Santander y Norte de Santander, en las primeras décadas del siglo XIX. Desde allí se expandió lentamente hacia el sur a lo largo de la cordillera Oriental. En el periodo entre 1840 y 1860 llegó hasta Cundinamarca, Tolima, algunas partes del Huila y el Cauca, y su producción estaba en manos de grandes terratenientes. [...] Hacia 1870, el café ya había llegado al departamento de Antioquia y al poco tiempo se convirtió en un cultivo de mucha acogida. En el periodo entre 1880 y 1910 , el café se extendió desde allí hacia el sur, hacia los departamentos de Caldas, Risaralda, Quindío y norte del Valle (del Cauca)." (Guhl, A., 2008:136)

La expansión que tuvo el café a principios del siglo XX implicó no sólo un desplazamiento de las zonas de producción, sino la presencia de nuevas formas de organización social y productiva, y se asoció a un crecimiento exponencial posterior a la Guerra de los Mil Días. De acuerdo con la Federación Nacional de Cafeteros de Colombia (FNC, 2015), el café Ilegó al Tolima en el año 1870 y apareció en los municipios de Venadillo, Santa Isabel y Rovira.

\footnotetext{
${ }^{21}$ Nombre dado en Colombia a la propiedad de uso agropecuario, en la que se asocia la vivienda con la producción agrícola. No necesariamente la finca significa que pertenezca a un minifundio. Pero cuando la finca es muy grande, perteneciente a un latifundio se llama 'hacienda'.

22 El cultivo para la provisión de alimento diario de la familia cafetera se denomina 'pancoger' 
En la región del Tolima se afianzaron grandes haciendas productoras de café, que son afectadas por el conflicto agrario que se gesta en la región. A partir de 1951 se intensifica el conflicto armado y en consecuencia, se abandonaron las grandes haciendas. A principios de los años sesenta los pequeños campesinos sin tierras presionaron la parcelación de los latifundios abandonados.

En el Tolima, el minifundio se constituyó en la característica dominante en gran parte del cultivo de café en la región. En 2014, el desarrollo de la caficultura en Tolima, estableció que el 97\% de su parque cafetero estaba tecnificado (CDCT, 2014).

Salamanca (2015) ha demostrado que existe una relación directa entre la producción en pequeñas parcelas y el enfoque en productos diferenciados de calidad, pues representan una opción viable para maximizar ingresos en el territorio disponible. El desarrollo de la caficultura del Tolima ha permitido la integración de pequeñas parcelas con el conocimiento trasmitido de tecnificación y especialización, y por tanto ha logrado que el café de esta región se haya destacado por su calidad.

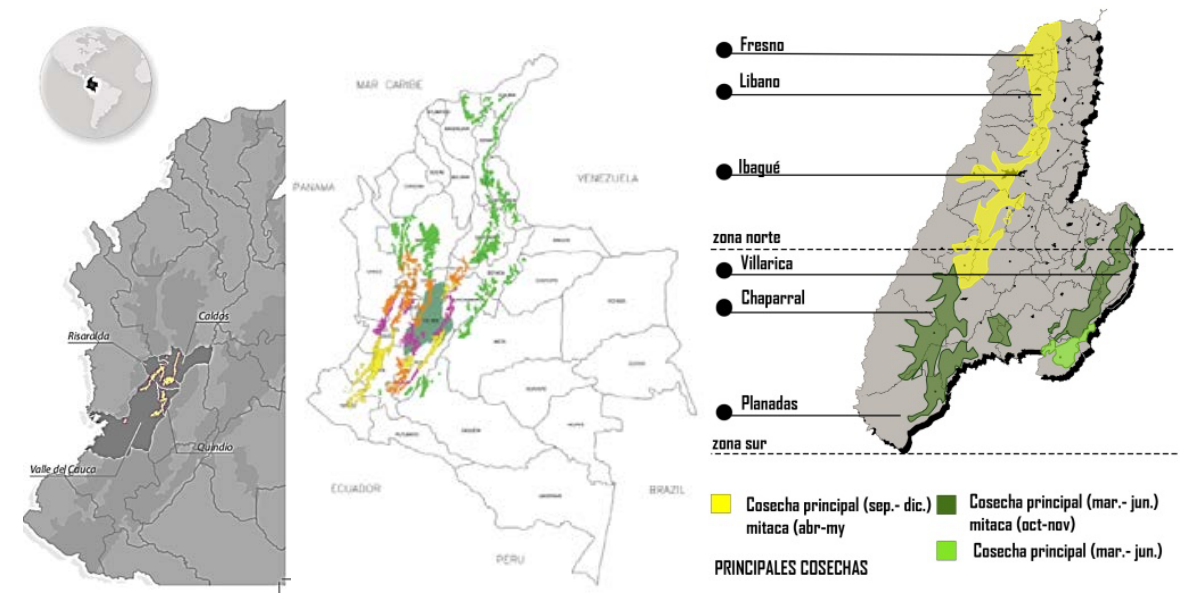

Fig. 02 Localización del Paisaje Cultural Cafetero (inscrito en la Lista de patrimonio mundial) y del Paisaje Cultural Cafetero del Tolima. Fuente: Federación Nacional de Cafeteros de Colombia; y elaboración de Haver Romero, Ana María Pérez y Louis Prieto.

\subsection{Localización}

Las características del café del Tolima provienen del rango de altitud entre 900 y $2100 \mathrm{msnm}$, en el que se localizan 38 municipios que conforman el paisaje productivo cafetero. Esto se refiere al localizado en 0252'59" y 05019'59" latitud norte, y 74024'18" y 76006'23" en la cordillera central, piedemonte oriental y occidental del rio Magdalena y valle del río Magdalena, que comparten cualidades derivadas de similares factores geográficos, de suelos, climáticos, de prácticas de cultivo y culturales.

Los municipios cafeteros del Tolima son: Alpujarra, Alvarado, Anzoátegui, Armero - Guayabal, Ataco, Cajamarca, Casabianca, Chaparral, Coyaima, Cunday, Dolores, Falan, Fresno, Herveo, Ibagué, Icononzo, Lérida, Libano, Mariquita, Melgar, Murillo, Natagaima, Ortega, Palocabildo, Planadas, Prado, Purificación, Rioblanco, Roncesvalles, Rovira, San Antonio, San Luis, Santa Isabel, Suárez, Valle de San Juan, Venadillo, Villahermosa y Villarrica.

La época de floración de la región cafetera de Tolima tiene disponibilidad de café durante todo el año, lo que define dos zonas con diferentes épocas de cosecha. La primera equivalente al $75 \%$ de los municipios de la región cafetera y que tiene su cosecha principal entre los meses de marzo y junio, y entre octubre y noviembre. La segunda zona comprende 
los municipios de Armero, Casabianca, Falan, Fresno, Herveo, Lérida, Libano, Murillo. Palocabildo, Santa Isabel, Villahermosa y tiene su cosecha principal entre septiembre y diciembre, y otra menor entre abril y mayo.

El paisaje cafetero de Tolima se conforma de suelos y condiciones climáticas que la diferencian de otras regiones productoras de café en Colombia. Las características del suelo y la distribución de las lluvias a lo largo del año generan una distribución equilibrada de la cosecha (Gómez, G., Caballero, R., Y Baldion, R., 1991). La precipitación varía entre 1.179 - 1.626 milímetros de lluvia al año; las temperaturas promedio oscilan entre $20.2^{\circ} \mathrm{C}$ en Planadas y $21.4^{\circ} \mathrm{C}$ en Fresno; las temperaturas mínimas están en el rango de 16.2 en Ibagué y 18.1 en Fresno, respecto a la temperatura máxima son del orden de $25^{\circ} \mathrm{C}$. Respecto al número de horas de Brillo Solar la variación existente es del orden de 1179 en Planadas y 1605 en el Líbano; para la lluvia las variaciones son de 1.647 mm/año en Ibagué y de 2.333 en Fresno. (FNC, 2015:26)

Los perfiles de suelo característicos del Tolima pertenecen a clasificaciones relativas a cenizas volcánicas, tobas andesíticas y granitos (feldespatos e intrusitas); areniscas, arenas, arcillas, esquistos y filitas. La combinación de estos elementos y su interacción con las características físicas y químicas de los suelos, que sumadas al uso del grano de café de la especie Coffea arabica, permiten que el café tenga una alta calidad. (FNC, 2015:27)

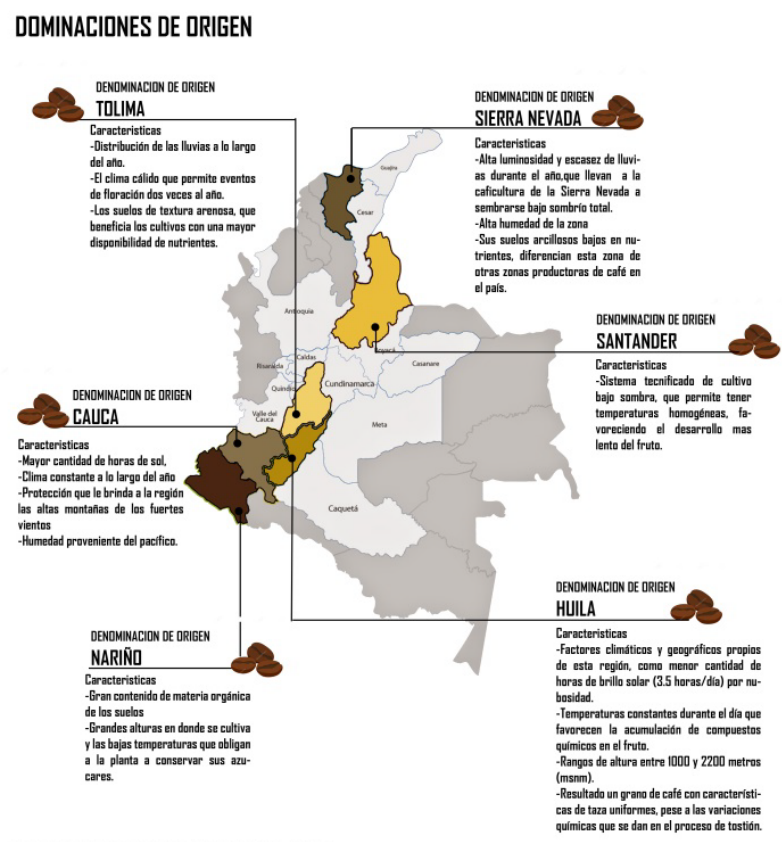

Fig. 03 Denominaciones de origen de la producción cafetera en Colombia. Fuente: Elaboración de Ana María Pérez y Louis Prieto

\section{LA ESTRUCTURA DEL PAISAJE CULTURAL}

\subsection{Descripción del paisaje y estructura geográfica}

El paisaje cafetero del Tolima se caracteriza por el cultivo del café en empinadas laderas, se conjuga la biodiversidad con la fisiografía de valles y laderas de pendientes suaves y escarpadas, hasta los glaciares y volcanes en las cumbres de la Cordillera Central y los bosques nativos y corredores biológicos, los cuales son considerados estratégicos para la conservación de la biodiversidad. Este territorio recoge un conjunto de ecosistemas en el cual coexisten complejos ecológicos y humanos que muestran una indivisibilidad compuesta por la unidad de cuencas con sus vertientes y planicies; la de los nevados; el páramo de Las Hermosas que bañan el ecosistema cafetero del Tolima y un subsistema de ciudades resultado de un proceso de población prehispánico. 
Conformada por la vertiente oriental de la Cordillera Central y la vertiente occidental de la cordillera Oriental, intensificada por la producción agrícola y ganadera, la región del Tolima se define por la cuenca hidrográfica del Río Magdalena, a la cual tributan los ríos Coello, Gualí, Recio, Saldaña y Totare. Las unidades acuíferas son resultado de la acumulación y la consolidación de sedimentos y depósitos fluvio-volcánicos, como el abanico de Ibagué, de Guamo y Espinal generados de la actividad de los volcanes Cerro Machín y el Nevado del Tolima (CARDER et al., 2002).

Una de las unidades ecológicas prioritarias de la región del Tolima es el Parque Nacional Natural de los Nevados, que comprende un área aproximada de 58.300 hectáreas y en las que la región del Tolima ocupa a los municipios de Ibagué, Anzoátegui, Santa Isabel, Murillo, Villahermosa, Casabianca y Herveo. El agua que nace y se regula en esta reserva y su zona de influencia abastece las necesidades de la población de la región. Por lo anterior, su protección y conservación se convierte en elemento clave para el desarrollo socio ambiental y en un eje articulador de las iniciativas de conservación regional. El PNN de los Nevados responde a un sistema o arco volcánico al cual pertenecen el Volcán Cerro Bravo al norte y el Volcán Cerro Machín al sur.

Los ecosistemas vinculados al paisaje cafetero tienen una gran importancia ambiental, con un gran número de bosques nativos y corredores biológicos, considerados estratégicos para la conservación de la biodiversidad global; según Conservation International ${ }^{23}$ esta región de los Andes suramericanos conforma uno de los biodiversity hotspots ${ }^{24}$ más importantes en el mundo, llamado Tropical Andes Hotspot. De acuerdo con CEPF 25 el hotspot de los Andes Tropicales son el hotspot ('punto crítico') más diverso del mundo, encabezando la lista de los 35 hotspots de mayor riqueza de especies y endemismos. Contiene alrededor de una sexta parte de toda la vida vegetal en el mundo, incluyendo 30.000 especies de plantas vasculares. Cuenta con la mayor variedad de especies de anfibios, aves y mamíferos, y ocupa el segundo lugar de Mesoamérica en cuanto a la diversidad de reptiles.

También se destaca por sus servicios ecosistémicos. Los Andes son considerados como las fuentes de agua de América del Sur, que sirven como fuente para los ríos Amazonas y Orinoco, (la tercera zona hidrográfica más grande del mundo por volumen de descarga). Las aguas andinas riegan las principales regiones agrícolas de América del Sur y proporcionan una importante fuente de poder por su capacidad de generación de energía hidroeléctrica (para una población estimada de 57 millones de habitantes de la región). Se calcula que los bosques andinos almacenan 5.400.000.000 de toneladas de carbono.

Los hotspots contienen a los KBA: Key Biodiversity Hostposts (Puntos críticos de Biodiversidad Clave) y corredores prioritarios, los cuales se refieren a las reservas delimitadas dentro del territorio biodiverso. Todos los KBA de la Cordillera Central de Colombia, representan los últimos restos de bosque montañoso andino en un paisaje en gran parte afectado por la expansión urbana, el pastoreo de ganado, y las plantaciones de café y procesos volcánicos que han transformado el paisaje hace mucho tiempo. Esta circunstancia hace que la protección de los KBA sea fundamental para la provisión de agua para el uso humano y la agricultura. (CEPF, 2015).

La ubicación en la zona de vida subandina (1.100-2.350 msnm), que hace parte de la cordillera de los Andes en su sector tropical (según la clasificación de zonas de vida de Holdridge (1992) por sus condiciones de “localización, relieve, clima y

${ }^{23}$ http://www.conservation.org/How/Pages/Hotspots.aspx

24 Un Biodiversity hotspot es una región biogeográfica con un depósito importante de biodiversidad amenazada por la acción humana. Para calificar como BH, una región debe contener al menos un 0,5\% o 1.500 especies de plantas vasculares como endémicas, y tiene que haber perdido al menos el $70 \%$ de su vegetación primaria. En el mundo, sólo 25 áreas califican bajo esta definición. Estos sitios soportan casi el $60 \%$ de las plantas del mundo, aves, mamíferos, reptiles y anfibios, con una muy alta proporción de especies endémicas. http://tmalliance.org/about/where-we-work/biodiversity-hotspot/
Critical
Ecosystem
Partnership
Fund. http://www.cepf.net/where we work/regions/south america/tropical andes/Pages/default.aspx 
suelos, esta región presenta un elevado número de hábitats de interés estratégico para la conservación de la diversidad biológica" (Rodríguez, D., Carranza, J., 2008).

La vegetación de la provincia puede dividirse en una de carácter andino en las tierras altas de las montañas y otra en las tierras bajas de los valles interandinos. En las montañas predominan la vegetación arbustiva y herbácea de los páramos, los bosques húmedos altoandinos y subandinos, las zonas lacustres, así como enclaves de vegetación seca altoandina y de vegetación semiárida subandina. (CORTOLIMA, 2014).

En las tierras bajas de los valles cálidos la vegetación está caracterizada por la selva húmeda, los bosque secos y de galería, los matorrales, las sabanas, los humedales y zonas lacustres. El clima es variado, con gran diversidad en la distribución y cantidad de las lluvias, por lo cual, presenta desde condiciones húmedas hasta áridas. Además, se encuentran todos los pisos térmicos: Cálido, Templado, Frio, Muy Frio, Extremadamente Frio y Nival. (LATORRE J., et al., 2014:23)

De esta provincia, se identifica principalmente con la zonificación de 3 distritos:

a) Distrito 'Bosques Altoandinos-Cordillera Central Vertiente oriental Complejo volcánico Ruiz - Santa Isabel'

b) Distrito 'Vegetación herbácea y arbustiva altoandina-Cordillera Central. Complejo volcánico Ruiz Santa Isabel ('Páramos Quindío')

c) Distrito 'Bosques Altoandinos-Cordillera Central Vertiente Occidental Complejo volcánico Ruiz - Santa Isabel’

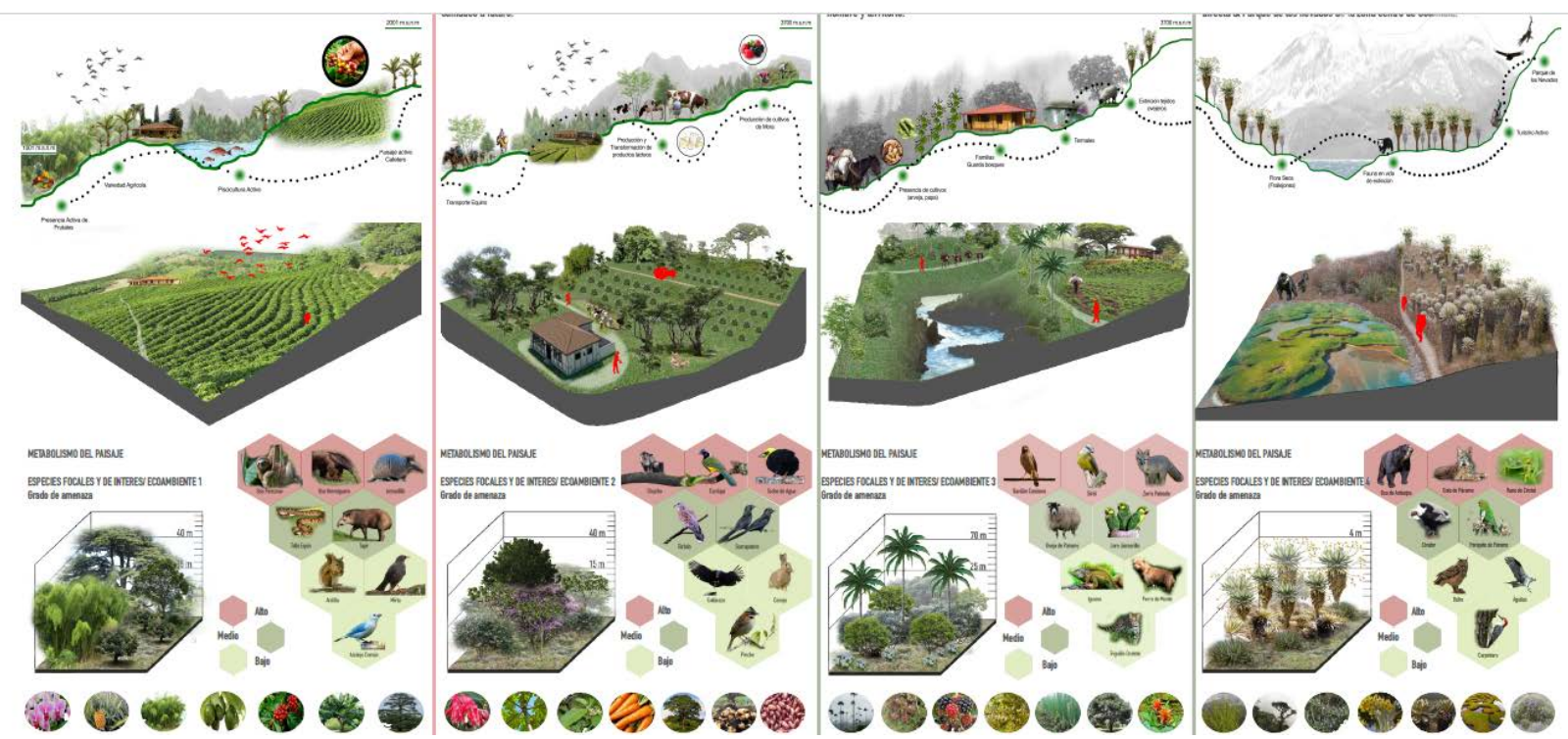

Fig. 04 Ecotopos del paisaje cultural cafetero del Tolima. De izquierda a derecha: Bosque de Galería, Bosque Montano y Submontano; Bosque Montano Alto y Páramo; Páramo y Súper Páramo. Fuente: Elaboración de Leidy Chica.

Deben señalarse las amenazas naturales que afectan la riqueza ambiental, productiva y cultural del paisaje. Entre las principales amenazas se han identificado las amenazas por movimientos en masa, inundación, amenazas volcánicas y sísmicas y riesgos por incendios forestales. Debido a que existen suelos residuales de espesor considerable, que conjuntamente con la pérdida de cobertura vegetal, la intensidad de lluvias y la pendiente, pueden generar deslizamientos. 
El territorio en donde se localiza el paisaje cafetero del Tolima tiene un alto riesgo sísmico, debido a la triple unión que ocurre en la esquina de la Placa tectónica Suramericana donde las placas de Nazca y Caribe convergen entre ellas.

Por otra parte, la actividad cafetera se ve afectada por fenómenos de variabilidad climática asociados al llamado 'fenómeno del Niño', ocasionado por el calentamiento diferencial del Océano Pacífico. Por ejemplo, como consecuencia de la ola invernal en 2010 y 2011, se desarrollaron problemas fitosanitarios: se propició el aumento a cifras históricas de los niveles de infección de la roya, se erosionaron los suelos cafeteros y disminuyó considerablemente la productividad de las plantaciones. (FNC, 2012)

Durante este periodo Cenicafé26, encontró que en la región las lluvias en las zonas cafeteras estuvieron 33\% por encima de la media histórica, llegando incluso en algunas regiones a incrementos entre $48 \%$ y $57 \%$; el brillo solar ${ }^{27}$ disminuyó en promedio un $13 \%$, lo que significa que los cafetales estuvieron sometidos durante 83 días sin recibir luz solar efectiva, condición vital para el desarrollo de las floraciones y la productividad de los cafetales; la temperatura: cayó $0,8^{\circ} \mathrm{C}$ con respecto a la media histórica, con variaciones regionales que alcanzaron incluso $1.3^{\circ} \mathrm{C}$ y que consecuentemente afectan significativamente el entorno productivo de la planta.

En cuanto a la amenaza volcánica de la ecorregión se relaciona con cinco volcanes activos pertenecientes al Complejo Volcánico Machín-Cerro Bravo, en el costado oriental de la Cordillera Central. A este sistema pertenecen el Volcán Nevado del Ruíz (VNR), el Volcán Nevado de Santa Isabel, el Volcán Cerro Machín (VCM), el Volcán Cerro Bravo y el Volcán Nevado del Tolima (VNT). De los anteriores, el VNR y el VCM, son los que mayor actividad presentan en su comportamiento con diferentes fluctuaciones en cuanto a la liberación de energía sísmica y demás parámetros geofísicos, geodésicos y geoquímicos (SGC, 2012).

El Tolima se vio afectado por el desastre de Armero en 1985, causado por el evento eruptivo del VNR, y que dejó profundos conflictos sociales y culturales, a raíz del arrasamiento de la ciudad de Armero por el lahar causando 27.000 víctimas. Por otra parte, debido a sus características, el VCM tiene el efecto potencial de un edificio explosivo. INGEOMINAS (2003) pronosticó impactos hemisféricos, regionales y locales de la erupción inminente del VCM y los comparó con los eventos de 1993 del Monte Pinatubo en Filipinas.

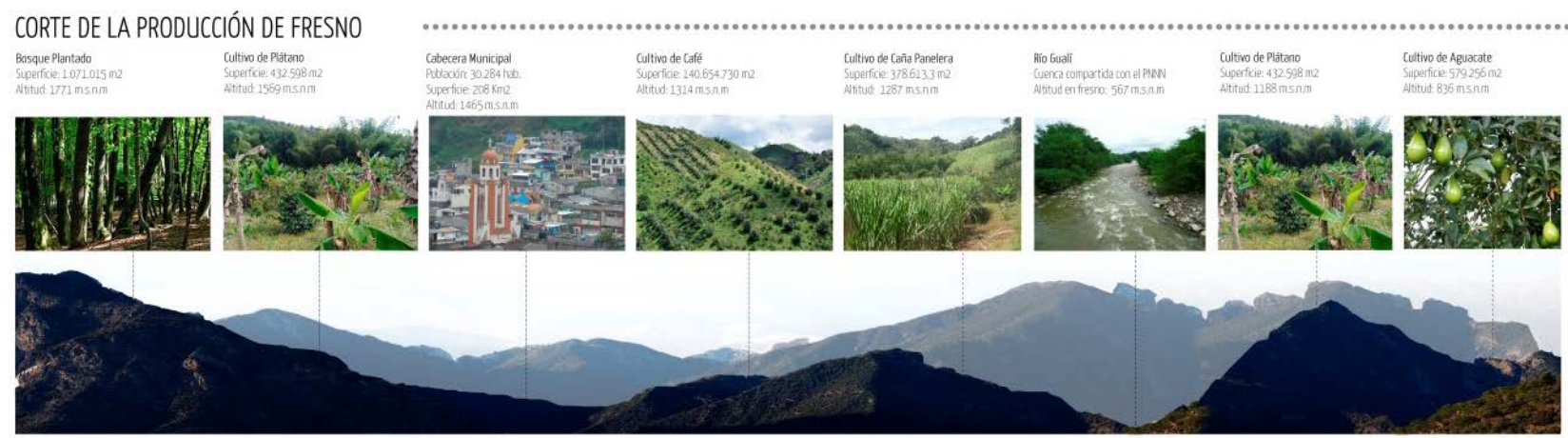

Fig. 05 Productividad del paisaje cultural cafetero en Fresno, Tolima. Fuente: Elaboración María Camila García.

\footnotetext{
${ }^{26}$ Centro Nacional de Investigaciones de Café. https://www.cenicafe.org/

27 Una mayor nubosidad asociada al nivel de lluvias registrado produjo un descenso en las horas sol y consecuentemente en la energía solar que requieren las plantas para los procesos de fotosíntesis. 


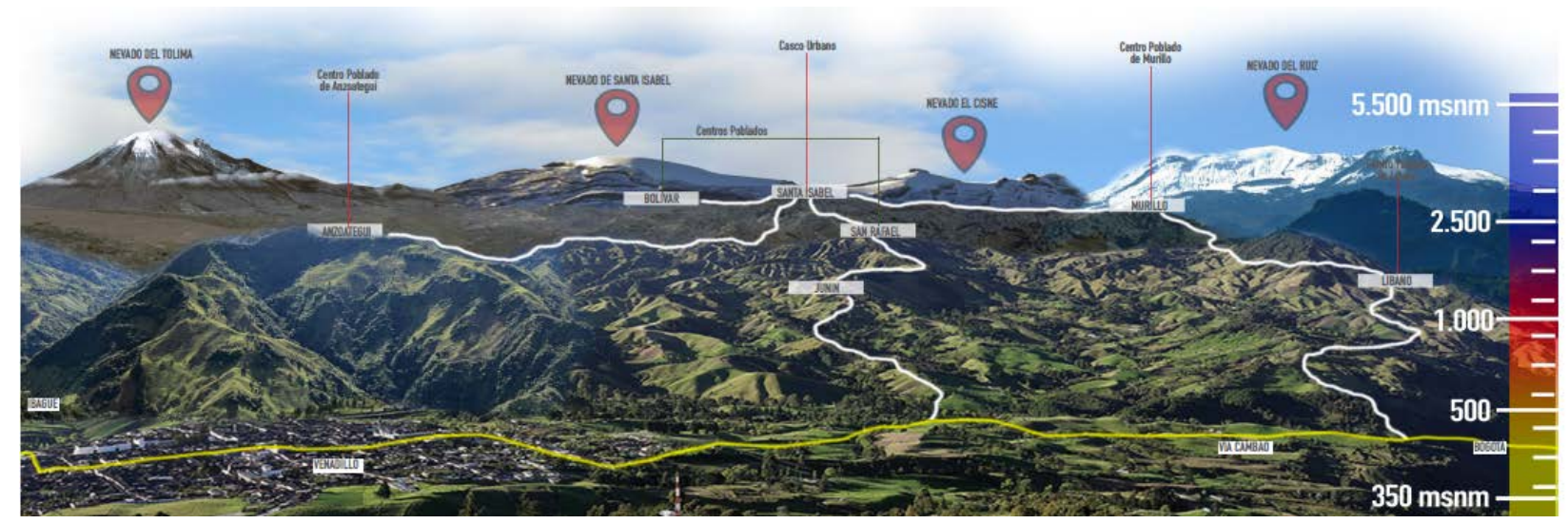

Fig. 06 Imaginario-representación del paisaje altitudinal de la Cordillera Central de los Andes vista desde el valle del Río Magdalena, en la región del Tolima. Fuente: Elaboración de Leidy Chica.

\subsection{Valores y atributos del paisaje cultural}

Basado en el expediente de nominación del Paisaje Cultural Cafetero de Colombia, los atributos del PCCC se referencian como componentes para la valoración del PCCT, en orden de establecer dónde y cómo están expresados territorialmente.

a) Café de montaña: "la caficultura del PCC se localiza principalmente entre los 1.000 y 2.000 m.s.n.m. [sic] en las laderas de las cadenas montañosas pertenecientes a las cordilleras Central y Occidental de los Andes colombianos. Debido a esa localización, es común referirse a ese cultivo como café de alta montaña. En particular, la zona principal del PCC se caracteriza por una altitud media que alcanza los 1.540 msnm". (MINCULTURA-FNC, 2009)

b) Predominio de café: expresa el uso preferencial de la tierra para el cultivo de café, antes que para otros cultivos.

c) Cultivo en ladera: representa la adaptación de los cultivos del café en zonas de alta pendiente, superiores al 25 \%, lo cual caracteriza la morfología particular del paisaje.

d) Edad de la caficultura: consiste en la renovación de plantaciones de café que permite mantener joven y vivo el paisaje. Este atributo posibilita la permanencia del PCC.

e) Patrimonio natural: la caficultura en el centro-occidente de Colombia se ubica en la ecorregión Andina Tropical; por sus condiciones de localización, relieve, clima y suelos, presenta un elevado número de hábitats de interés estratégico para la conservación de la diversidad biológica.

f) Disponibilidad hídrica: se refiere a la alta presencia de unidades prioritarias para la retención y regulación del agua. La oferta de agua es determinante en la cosecha del café. 
g) Institucionalidad cafetera y redes afines: se refiere a la existencia de redes institucionales y económicas que inciden en el funcionamiento y dinámica del PCC. Son la garantía de la sostenibilidad del paisaje como sitio patrimonial.

h) Patrimonio arquitectónico: el PCC posee un patrimonio que ha sido creado por los pobladores de la zona. Se trata de la arquitectura regional de bahareque, y la utilización intensiva de la guadua (Guadua angustifolia) que se expresa en los saberes tradicionales del diseño y construcción de sus viviendas.

i) Patrimonio arqueológico: se conoce de la presencia cultural del hombre desde hace diez mil años en el actual Eje Cafetero. Aparte de los vestigios arqueológicos de orfebrería y cerámica, a esos antepasados les debemos la domesticación de animales y plantas alimenticias, e incluso técnicas agrícolas que quizá puedan rastrearse en la actual producción de café.

j) Poblamiento concentrado y estructura de la propiedad fragmentada: otro de los rasgos característicos de la caficultura del PCC es la predominancia de la pequeña unidad productiva. En efecto, el tamaño promedio de la finca cafetera en el área principal del paisaje es de apenas 4.6 hectáreas, de las cuales 2.6 hectáreas se encuentran cultivadas con café. Esta estructura de tenencia de la tierra, denominada de división parcelaria 'menuda', es evidencia de una significativa redistribución o democratización de la propiedad rural. Esta característica se asocia al proceso histórico de colonización de la zona en la segunda mitad del siglo XIX.

k) Influencia de la modernización: comprende la adaptación del paisaje a las condiciones de la vida moderna, como la infraestructura de vías de comunicación, servicios públicos, salud y educación.

I) Patrimonio urbanístico: la forma de nuestros pueblos se basa en el modelo hispánico del trazado en cuadrícula. Este tipo de estructuras urbanas, en contraposición con el relieve, las calles de gran pendiente y las manzanas ortogonales, es una muestra de la adaptación de la cultura cafetera a las condiciones ambientales particulares de la topografía quebrada, que da como resultado las estructuras urbanas con formas de damero en ladera.

m) Tradición histórica en la producción de café: hace referencia a la persistencia del cultivo de café y la resistencia al cambio en el uso del suelo, a pesar de los ciclos y crisis económicas que afectan la producción cafetera.

n) Minifundio cafetero como sistema de propiedad de la tierra: la prevalencia del minifundio es otro elemento que configura el paisaje cafetero.

o) Cultivos múltiples: es la multiplicidad de cultivos que conforman una 'colcha de retazos', elemento característico del PCC.

p) Tecnologías y formas de producción sostenibles en la cadena productiva del café: este atributo muestra las condiciones para producir café de manera sostenible y cómo la comunidad cafetera ha adaptado su forma de trabajo tradicional para lograr mejores y modernas condiciones de producción, con menos impactos ambientales. (MINCULTURA-FNC, 2014:13). 
Adicionalmente, la investigación debe dar cuenta de atributos que no están desarrollados en el expediente inscrito del PCCC:

a) Conflicto armado. En la región del Tolima tuvo origen el conflicto armado, a raíz de un movimiento agrario y la represión por parte del Ejército, causó el desplazamiento de la población y la caída del precio del café. (Molano, A., 2015)

b) Denominación de Origen. Las propiedades de calidad físico-químicas, de suelos, manejo agronómico del cultivo del café, ligadas a las condiciones climáticas y altitudinales de la zona ecuatorial colombiana, determinan una variación de alta calidad para la denominación de origen "Café del Tolima".

c) Cafés especiales: los programas de cafés especiales que han desarrollado los cafeteros de la región promovidos por la Federación y por diferentes entidades nacionales e internacionales. Esto se expresa en un amplio número de marcas en el mercado que tiene la denominación de origen como una de sus líneas especiales. De acuerdo con indicadores de sostenibilidad, que incluyen diferentes sellos de certificación y verificación asociados con prácticas sostenibles, son validados por organizaciones internacionales especializadas.

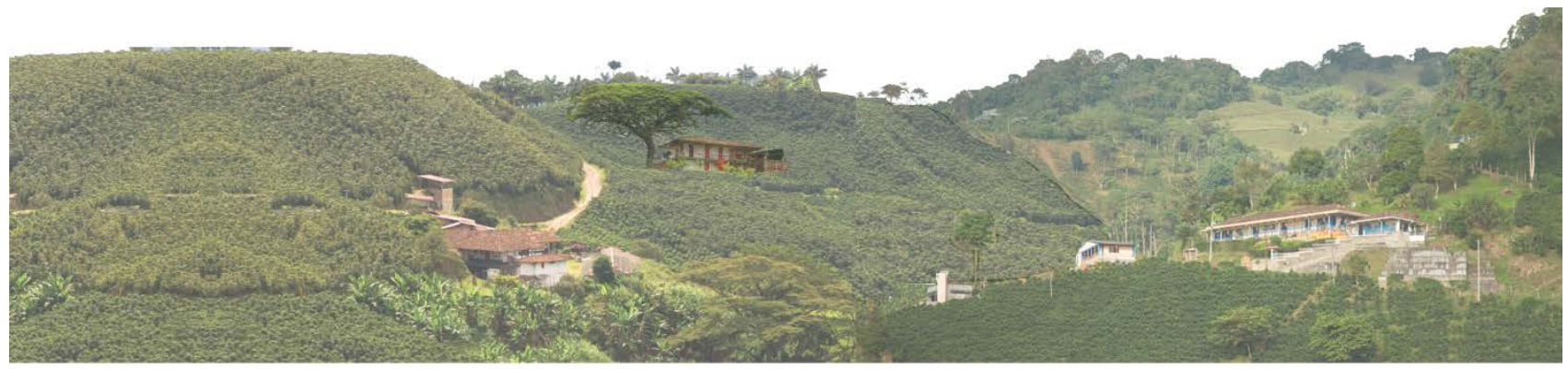

Fig. 07 Imaginario fotográfico del Paisaje cultural cafetero de Planadas, Tolima. Fuente: Elaboración de Javier Londoño y Lizeth González.

\section{LAS CONCLUSIONES: RETOS Y ESTRATEGIAS PARA EL MANEJO}

Aunque el proyecto aún requiere un mayor desarrollo de los resultados y contrastar lo propuesto en evidencias de un modelo cartográfico de información geográfica en construcción, las recomendaciones deben dirigirse a la fundamentación de estrategias de manejo de las problemáticas detectadas.

Las estrategias sobre ordenamiento territorial y de manejo paisajístico, deben guardar relación con los riesgos de desastre en el sitio y en su entorno inmediato. En Colombia, existe la necesidad de integrar el patrimonio en los planes urbanísticos o regionales existentes y en proyectos de planificación concretos al tratar de la integración de los planes de gestión del riesgo de desastres con los demás planes. (Velandia, C., 2016).

Para tal fin, se requiere de la armonización de los aspectos patrimoniales con el ordenamiento territorial de los paisajes culturales (DUIS, U., et al. 2010). Mediante un ejercicio de diagnóstico del paisaje - conocimiento interno y externo, la propuesta de construcción de programas, proyectos y la articulación con el ordenamiento territorial, pretende la organización sostenible del paisaje a partir de la identificación de las potencialidades y amenazas que posee el paisaje, las cuales son definidas básicamente por las características ambientales, culturales y socioeconómicas del territorio. Las categorías para la elaboración del diagnóstico son:

- La oferta: definida como la capacidad y potencial para producir bienes y servicios ambientales, culturales, institucionales; 
- La demanda: representada por el uso actual y requerimientos de las poblaciones humanas sobre el ambiente biofísico y cultural y;

- Los conflictos: generados por incompatibilidades entre la oferta y la demanda que se manifiestan en destrucción, degradación o sobreutilización de los componentes del paisaje.

Se coincide con la metodología para caracterizar con un enfoque participativo el Paisaje Cultural integrando las dimensiones ecológica, social, biofísica, productiva, económica, cultural y legal; proponer escenarios para el uso y manejo sostenible a nivel de sus sectores; consolidar y difundir una base de información territorial del paisaje haciendo partícipes a las comunidades. (Duis, U., et al. 2010:14).

Con base la integración de la metodología, sobre una estructura operativa de unidades de paisaje, se podrá complementar la política para el manejo del ordenamiento territorial y de manejo paisajístico, en la medida que estas unidades estén cualificadas por los lineamientos del paisaje cultural, y de ellas se precisen las condiciones y las acciones específicas para la preservación de las áreas agrícolas y de cultivo de café, los intercambios socioculturales y económicos, las manifestaciones culturales y la conservación de los ecosistemas.

En cuanto a las estrategias para la apropiación del patrimonio, la protección del paisaje cultural debe favorecer el mejoramiento de las condiciones de vida de las poblaciones locales a partir de modelos de desarrollo sostenible. La cohesión del tejido social a desarrollar mediante los procesos de educación y reconciliación para la paz, es la menos avanzada desde el enfoque patrimonial, ya que los procesos de apropiación son complejos de reconocer y desarrollar. Es la única forma que puede garantizarse la transmisión de los valores, y de potenciar la sensibilidad colectiva de las comunidades que pueblan y trabajan el paisaje cultural.

Al paisaje, como producto social transformado por el trabajo colectivo, puede descifrarse en sus componentes por la arqueogeografía, y el trabajo antropológico y etnográfico complementario con las comunidades, para interpretar la carga simbólica del paisaje cultural.

Por otra parte, las estrategias para la gestión del riesgo deben aportar en cuanto al dimensionamiento de su impacto sociocultural y económico, y las posibles integraciones con el ordenamiento territorial. En todos los casos, se valoran las amenazas más comunes, como los deslizamientos y la remoción en masa. En el caso de Colombia, la amenaza volcánica así como la sísmica, es elevada (World Bank, 2014)

De acuerdo con el Manual de referencia para la gestión del riesgo de desastres (UNESCO, 2010) ¿Cómo se vincula un plan de Gestión de Riesgo y Desastres (GRD), al plan de manejo del bien patrimonial? Esto no es posible si no se considera la amenaza volcánica como prioritaria y si no se identifican los enlaces naturaleza cultura del patrimonio con el riesgo de amenaza volcánica.

En relación a la estrategia de la variabilidad climática y la sostenibilidad ambiental, en materia de adaptación al cambio climático existen antecedentes desde la fundación del Fondo de adaptación para el cambio climático ${ }^{28}$ por parte del Gobierno de Colombia, que ha logrado el financiamiento internacional con USAID ${ }^{29}$, y con el Plan de las Naciones Unidas para el Desarrollo (PNUD) ${ }^{30}$. La FNC ha desarrollado la política de sostenibilidad en acción, para enfrentar medidas

${ }^{28}$ Ministerio de Hacienda, Gobierno de Colombia. http://sitio.fondoadaptacion.gov.co/

29 US Agency forlnternational Development https://www.usaid.gov/es/where-we-work/latin-american-and-caribbean/colombia

30 www.pnud.org.co/cambioclimatico/ Consultado 1 de octubre de 2015. 
sostenibles de productividad ante el cambio climático, intensificar la promoción y aplicación de estrategias y acciones de “Caficultura Climática Inteligente" (Uribe, S., Velandia, C., 2013)

Para la construcción de la estrategia para la paz y la reconciliación, con la firma del Acuerdo de Paz con las Fuerzas Armadas Revolucionarias de Colombia (FARC) en 2016, se parte de uno de los puntos principales fue el Acuerdo sobre "Política de Desarrollo Agrícola Integral Hacia un Nuevo Campo Colombiano: Reforma Rural Integral (RRI)". Este acuerdo establece las bases para la transformación del campo y crea las condiciones de bienestar y buena vida para la población rural. Busca erradicar la extrema pobreza rural y reducir la pobreza rural en un $50 \%$ en un período de 10 años, promoviendo la igualdad, cerrando la brecha entre el campo y la ciudad, reactivando el campo y en particular el desarrollo de la agricultura campesina, familiar y comunitaria. ${ }^{31}$

Las estrategias mencionadas, deben orientarse a la conservación y promoción del patrimonio material e inmaterial del paisaje cultural y articularlo al desarrollo regional, mediante su identificación, estudio y manejo y el desarrollo humano sostenible. En este sentido, las soluciones deben intensificar el desarrollo de proyectos productivos que brinden perspectivas de desarrollo dentro de la actividad cafetera, metodologías adecuadas a las condiciones del campo que promuevan la formación para el trabajo y el arraigo en las zonas cafeteras y el diseño de programas de seguridad social que propicien condiciones de retiro justo para los caficultores mayores.

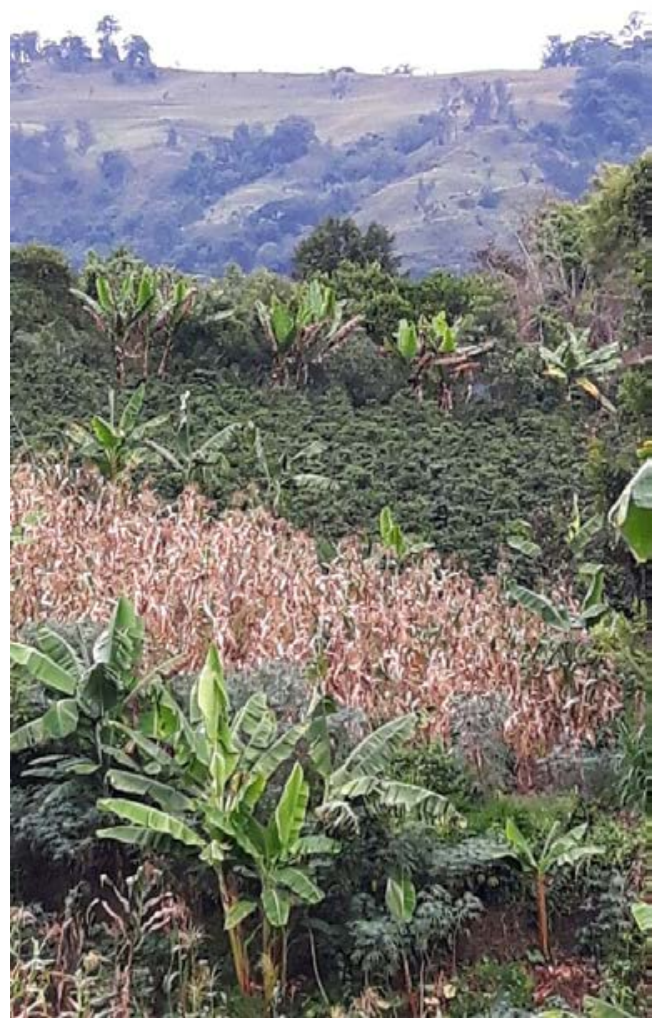

Fig. 08 Paisaje cafetero del corregimiento de Tapias, municipio de Ibagué, Tolima, Colombia. Fotografía del autor.

${ }^{31}$ http://www.altocomisionadoparalapaz.gov.co/Documents/informes-especiales/abc-del-proceso- de-paz / index.html) en el marco del posconflicto 


\section{BIBLIOGRAFÍA}

ANSCHUETZ, K. et al. (2001). An Archaeology of landscapes: Perspectives and Directions. En Journal of Archaeological Research, 9 (2), p.157-211.

BANDARIN, F., et al. (2009). World Heritage Cultural Landscapes: A Handbook for Conservation and Management. París: UNESCO World Heritage Centre. p.3.

BONNEMAISON, Joel (2005) Culture and Space: Conceiving a New Geography. New York: Tauris.

CARDER et al. (2002). Ecorregión Eje Cafetero: Un Territorio de Oportunidades. Pereira.

COMITÉ DEPARTAMENTAL DE CAFETEROS DE TOLIMA (CDCT). (2014) Informe social 2014. Ibagué: CDCT.

CORTOLIMA (2014). Atlas Ambiental del Tolima. Ibagué: El Nuevo Dia. https://www.cortolima.gov.co/sites/default/files/atlas/\#p=1

COSGROVE, D. (1985). Prospect, perspective and the evolution of the landscape idea. En Transactions of the Institute of British Geographers. 10 (1), pp.45-62.

CRIADO-BOADO, F. (2014) Archaeologies of Space: an Inquiry into Modes of Existence of XScapes". En K. Kristiansen et al. (Eds.) Paradigm Found. Archaeological Theory - Present, Past and Future. Essays in Honour of Evzen Neustupný, pp.61-83. Oxford: Oxbow.

CRITICAL ECOSYSTEM PARTNERSHIP FUND (CEPF) (2015). Ecosystem Profile. Tropical Andes Biodiversity Hotspot. Critical Ecosystem Partnership Fund.

DEETZ, J. (1990). "Landscapes as cultural statements”. En W. Kelso y R. Most (Eds.) Earth Patterns: Essays in Landscape Archaeology, pp. 1-4. Charlottesville: University Press of Virginia.

DUIS, U. et al. (2010). Guía para la Integración del Plan de Manejo del Paisaje Cultural Cafetero en el Ordenamiento Territorial. Pereira: Universidad Tecnológica de Pereira.

FALS, O., et al. (1988). La insurgencia de las provincias. Hacia un nuevo ordenamiento territorial para Colombia. Universidad Nacional de Colombia. Bogotá: Siglo XXI Editores.

FEDERACIÓN NACIONAL DE CAFETEROS DE COLOMBIA (FNC) (2012). Sostenibilidad en Acción 2011. Bogotá: FNC. (2015). Denominación de origen Café del Tolima. Documento de trabajo. Bogotá: FNC.

FUNDACIÓN ERIGAIE (2010) Lista Indicativa de Patrimonio Cultural y Natural de Colombia. Bogotá: Fundación Erigaie. GÓMEZ, G., CABALLERO, R., y BALDION, R. (1991). Ecotopos cafeteros de Colombia. Bogotá: FNC.

GUHL, A. (2008). Café y cambio de paisaje en Colombia, 1970-2005. Medellín: EAFIT- Banco de la República.

GUHL, E. (1988). La geografía y el ordenamiento territorial. En O. Fals Borda, La insurgencia de las provincias Hacia un nuevo ordenamiento territorial para Colombia. Pp. 143-236. Bogotá: Universidad Nacional de Colombia - Siglo XXI editores.

HIRSCH, E. y O'HANLON, M. (Eds.) (1995). The Anthropology of Landscape: Perspectives on Place and Space. Oxford: Clarendon Press. 
HOLDRIDGE, L. (1992) Life Zone Ecology. San José: Tropical Science Center.

INGEOMINAS (2003). Memoria Explicativa del Mapa de Amenaza Volcánica del Cerro Machín. Bogotá: Ingeominas. INGOLD, T. (1993). The temporality of landscape. En World Archaeology 25, pp.152-174.

LARSEN, P., WIJESURIYA, G. (2015). Interrelaciones entre naturaleza y cultura en el Patrimonio Mundial. En Patrimonio Mundial - Enlaces Naturaleza Cultura. Abril $n^{\circ} 75$. Paris: Centro de Patrimonio Mundial. Unesco. http://whc.Unesco.org/en/review/75

LATORRE, J., et al. (2014). Condición de las Unidades Ecobiogeográficas Continentales y Sistema Nacional de Áreas Protegidas en Colombia. Bogotá: Parques Nacionales Naturales de Colombia.

MINISTERIO DE CULTURA-FEDERACIÓN NACIONAL DE CAFETEROS DE COLOMBIA (2009). Plan de Manejo y Protección del Paisaje Cultural Cafetero. Bogotá: MINCULTURA-FNC.

MOLANO, A. (2015). Fragmentos de la Historia del Conflicto Armado (1920-2010). Bogotá: Espacio Crítico.

NORTON, W. (1989). Explorations in the Understanding of Landscape: A Cultural Geography. Nueva York: Praeger.

RODRÍGUEZ, D., CARRANZA J. (2008). El patrimonio natural del Paisaje Cultural Cafetero en Risaralda (Colombia), En, J. Osorio y A. Acevedo (coords.), Paisaje Cultural Cafetero: Risaralda Colombia. Pereira.

RÖSSLER, M. (1993). "The integration of cultural landscapes into the world heritage, conserving outstanding cultural landscapes, Protecting outstanding cultural landscapes." En, World Heritage Newsletter, 1-3.p.25. Unesco: World Heritage Centre. http://whc.unesco.org/en/newsletter/1/

RÖSSLER, M. (2003). "Linking Nature and Culture: World Heritage Cultural Landscapes". En, World Heritage Papers 7. p. 10-15. Unesco: World Heritage Centre. http://whc.unesco.org/documents/publi_wh_papers_07_en.pdf

RÖSSLER, M. et al. (2009). "World Heritage Cultural Landscapes: A Handbook for Conservation and Management". En, World Hertage Papers 26.p.15-135 Unesco: World Heritage Centre. http://whc.unesco.org/documents/publi_wh_papers_26_en.pdf

RÖSSLER, M., TORNOUX M. (2013). Patrimonio Mundial nº 69. París: UNESCO World Heritage Centre. p.15.

SABATÉ, J. (2010). De la preservación del patrimonio a la ordenación del paisaje: intervenciones en paisajes culturales (Europa - Latinoamérica). En Labor \& Engenho. (4) 1, pp.10-25. Campinas. http://periodicos.sbu.unicamp.br/ojs/index.php/labore/article/view/1686

SALAMANCA, J. (13 de Septiembre de 2015). Pistas para la Alta Productividad en Fincas Pequeñas. http://www.javeriana.edu.co/pesquisa/?p=4145

SERVICIO GEOLÓGICO COLOMBIANO (SGC). (2012). Informe de Actividad del Complejo Volcánico Cerro Bravo-Cerro Machín. Enero. Manizales: SGC.

TUAN, Yi Fu (1977). Space and Place: The Perspective of Experience. Minneapolis: University of Minnesota Press.

UNESCO (2008). Directrices Prácticas para la aplicación de la Convención de Patrimonio Mundial. París: Centro de Patrimonio Mundial. 
UNESCO (2010). Managing Disaster Risks for World Heritage. Paris: World Heritage Centre.

URIBE, S., VELANDIA, C. (2013). Naturaleza, Cultura y Productividad. Gestión del Paisaje Cultural Cafetero de Colombia. En N. NIÑO, y J. SALDAÑA, (coord.). Tercer Congreso Pydes 2013. México D.F.: Universidad Autónoma de GuerreroEditorial Praxis.

VELANDIA, C. (2016). El Paisaje Cultural Cafetero y su articulación con el ordenamiento territorial. En: F. RINCÓN, (Coord.) La caficultura como patrimonio cultural, social y productivo. Paisaje Cultural Cafetero de Colombia: cinco años como patrimonio mundial. Gestiones interinstitucionales en el marco del plan de manejo del Paisaje Cultural Cafetero entre 2011 y diciembre 2015. Manizales: MINCULTURA-FNC.

WORLD BANK (2014) Insights in DRM (Disaster Risk Management) A Practitioner's Perspective on Disaster Risk Management in Latin America \& the Caribbean. En Insights n8 p.1. www.worldbank.org/lcrdrm/insights

\section{Fuentes electrónicas:}

http://www.visiontolima2025.org/index.php/ideas-fuerza/macroproyectos/60-macroproyectos

(Consulta: 02/02/2018)

https://www.federaciondecafeteros.org/static/files/Tolima3.pdf (Consulta: 05/02/2018)

https://tolima.federaciondecafeteros.org/fnc/nuestro comite/category/118 (Consulta: 20/02/2018)

https://issuu.com/tolimacafetero/docs/tolima cafetero_224ok/2 (Consulta: 20/02/2018)

http://www.altocomisionadoparalapaz.gov.co/Documents/informes-especiales/abc-del-proceso- de-paz / index.html) en el marco del posconflicto (Consulta: 20/02/2018) 